\title{
ADVANCES IN ATMOSPHERIC TEMPERATURE PROFILE MEASUREMENTS USING HIGH SPECTRAL RESOLUTION LIDAR
}

\author{
Ilya I. Razenkov, Edwin W. Eloranta
}

\author{
University of Wisconsin-Madison, 1225 W. Dayton St., Madison, WI, 53706,USA, razenkov@wisc.edu
}

\begin{abstract}
This paper reports the atmospheric temperature profile measurements using a University of Wisconsin-Madison High Spectral Resolution Lidar (HSRL) and describes improvements in the instrument performance. HSRL discriminates between Mie and Rayleigh backscattering [1]. Thermal motion of molecules broadens the spectrum of the transmitted laser light due to Doppler effect. The HSRL exploits this property to allow the absolute calibration of the lidar and measurements of the aerosol volume backscatter coefficient. Two iodine absorption filters with different line widths are used to resolve temperature sensitive changes in Rayleigh backscattering for atmospheric temperature profile measurements.
\end{abstract}

\section{INTRODUCTION}

The atmospheric temperature is an important parameter characterizing the state of the atmosphere. Accurate temperature profiles are required for modeling atmospheric processes as well as for calibration of remote sensing instruments. Radiosonde measurements are usually available only twice a day and with very sparse ground coverage. Lidars are capable of measuring the atmospheric temperature with a relatively high spatial and temporal resolution. Several investigators have used High Spectral Resolution Lidar for temperature profiling $[2,3]$. The reported techniques have low sensitivity to air temperature and the used instruments greatly exceed the eye-safe limit on the transmitted laser power.

The standard HSRL calibration requires the atmospheric temperature and pressure profile and typically uses radiosonde measurements. In order to enable temperature profile measurements, HSRL receiver has two iodine absorption filters with different absorption line widths [4]. This measurement capability makes the lidar intrinsically calibrated. The HSRL temperature measurement technique requires superior instrument performance. In order to achieve good measurement accuracy, the instrument has several modifications, which have improved instrument performance and data quality for standard HSRL measurements.

\section{HIGH SPECTRAL RESOLUTION LIDAR SYSTEM}

The simplified schematic of the HSRL lidar is shown in Figure 1. For a more detailed description of the instrument see "High Spectral Resolution Lidar at the University of Wisconsin-Madison" [5]. The transmitter uses a diode pumped $\mathrm{Nd}$ :YAG laser operating at $4 \mathrm{kHz}$ repetition rate. The average transmitted power is 200mW @ $532 \mathrm{~nm}$. The host laser cavity is injection seeded with the single mode distributed feedback diode (DFB) seed laser (1064nm). Active control loop is implemented to lock the seed laser frequency to the center of iodine absorption line \#1109. The frequency locking setup uses a $100 \mathrm{~mm}$ long iodine absorption cell and two detectors. The frequency locking accuracy is around $\pm 15 \mathrm{MHz}$ with a frequency jitter of around $\pm 15 \mathrm{MHz}$ amplitude.

A portion of the laser light is picked off from the beam by a wedged beamsplitter for frequency locking, laser spectrum quality control, and for the interferometer. The broadband beam splitter $(\mathrm{R} / \mathrm{T}=65 / 35 \mathrm{~S})$ is used as a transceiver switch. Then, the beam is further expanded by 20x DallKirkham telescope ( $400 \mathrm{~mm}$ aperture) to the eyesafe level and transmitted into the atmosphere. The telescope is shared between the transmitter and the receiver. The received light is split, filtered, and detected by the three channels: Combined, Molecular I2, and Molecular I2A. Signal in the combined channel contains photons scattered by both aerosols (Mie scattering) and by molecules (Rayleigh scattering). The first standard iodine filter (1.8 GHz Iodine line width) absorbs most of the aerosol signal photons (>99.98\%) 
allowing around $25 \%$ of the molecular signal photons through this cell. The second iodine absorption filter contains an argon buffer gas, which broadens the iodine absorption line to 2.85 GHz. It allows around $50 \%$ of the first stage filtered molecular signal photons through this cell providing approximately equal count rate in both molecular channels.

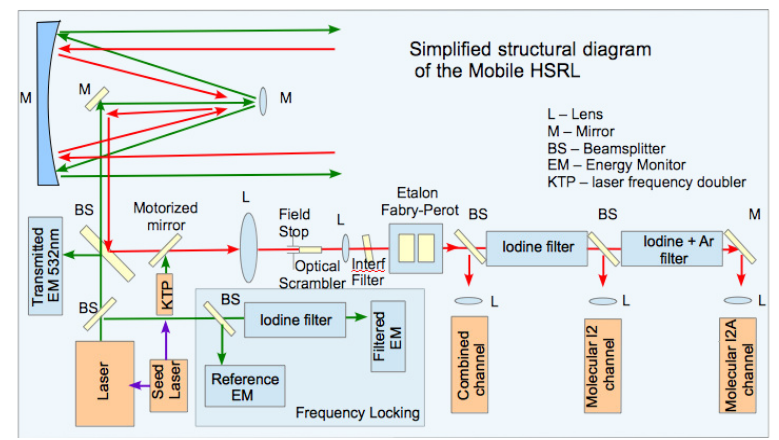

Figure 1. A simplified structural diagram of HSRL.

The argon-broadened filter minimum transmission in the center of the 1109 line is $\sim 0.1 \%$ and cannot provide sufficient rejection of aerosol signal. For that reason a serial arrangement of the filters is used. Both absorption filters contain a fixed amount of iodine to provide stable filter properties. Heaters maintain all the iodine in the gaseous phase and ensure that no iodine condensation occurs on the windows of the cell. The cells temperature is around 33 degrees $C$. The pressure and the iodine amount in the argon gas buffered iodine cell were optimized to provide the instrument sensitivity of the signal to air temperature and transmission. The optimum pressure is found to be at around 110 Torr of the argon gas pressure providing the instrument temperature sensitivity coefficient $\frac{d R}{d T}$ around $0.145 \% / \mathrm{K}$.

In the previous instrument configuration, the difference in optical alignment of the two molecular channels introduced a range dependent differential geometry error in the temperature profile measurements. In order to eliminate this effect, a $25 \mathrm{~mm}$ long straight multimode optical fiber is installed behind the field stop with the core size equal to the field stop aperture $(300 \mu \mathrm{m})$. The multimode fiber (optical scrambler) changes the intensity distribution of the received light and decreases the range dependency of the signals. It also decreases errors associated with non-uniform sensitivity of the detectors across the sensor area.

In order to measure the channels' transmission spectrum functions and the relative gain of the channels, we implemented seed laser frequency scans across the receiver bandwidth. The 95\% duty cycle light pulses $(\sim 237 \mu$ s pulse width) of the frequency doubled seed laser light $(532 \mathrm{~nm})$ with a large signal levels significantly improved the accuracy of the measured channels' transmission spectrum functions. Previously, we used short ( $\sim 50 \mathrm{~ns})$ pulses from the host laser at $4 \mathrm{kHz}$ pulse repetition rate. The seed laser scans also decreased the uncertainty in the laser output frequency and a laser spectral width. The newly measured channels' transmission functions do not require any adjustments for standard HSRL measurements. The temperature profile offsets due to scan-to-scan variations became smaller by approximately four times.

During instruments' operation the gain of the channels can change. In order to correct for these changes, we introduce high intensity green LED pulses ( $30 \mu$ s wide) into the receiver field-of-view $180 \mu \mathrm{s}$ after the transmitted laser pulse. The ratio of the signals in molecular channels from the LED accounts for the relative gain change in the two channels: changes in iodine filter transmission and detector gain changes.

\section{ATMOSPHERIC TEMPERATURE MEASUREMENT PRINCIPLES}

The ratio $R$ of the signals of the two molecular channels is a function of the atmospheric temperature and pressure. The received signals in the channels Molecular I2 $\left(N_{m, 1}\right)$ and Molecular $I 2 A\left(N_{m, 2}\right)$, for $i=1$ or 2 are:

$$
N_{m, i}(z)=N_{0}(z) f_{m, i}(z)+N_{a}(z) C_{a m, i}(z),
$$

where,

$f_{m, 1}(z)=\int \xi_{1}(v) F_{1}(v) \mathcal{R}(T(z), P(z), v) d v$, $f_{m, 2}(z)=\int \xi_{2}(v) F_{1}(v) F_{2}(v) \mathcal{R}(T(z), P(z), v) d v ，$ and $\int \mathcal{R}(T(z), P(z), v) d v=1, N_{0}(z)$ - number of photons received from a distance $z ; \xi_{1}(v), \xi_{2}(v)$ the channels' gains; $v$ - frequency; $F_{1}(v), F_{2}(v)-$ transmission spectrums of a standard iodine and broadened iodine filters correspondingly; $\mathcal{R}(T, P, v)$ - spectrum of molecular return from 
Tenti's s6 model; $T(z), P(z)$ - atmospheric temperature and pressure; $N_{a}(z) C_{a m, i}(z)$ crosstalk terms allowing a leakage of the aerosol photons into the molecular channels, which are significant only for dense clouds and can be omitted for clear sky conditions. The $N_{a}(z)$ and $C_{a m, i}(z)$ are derived from the standard HSRL inversion technique which uses the signal from the Combined channel. The channels' transmission spectrum functions $\xi_{1}(v) F_{1}(v)$, $\xi_{2}(v) F_{1}(v) F_{2}(v)$ are measured by performing a seed laser frequency scan across the receiver bandwidth. This provides the filter spectral bandpass and the relative sensitivity of the channels. The ratio of the signals in the two channels is the function of temperature and pressure:

$$
R(z)=\frac{N_{m, 1}(z)-N_{a}(z) C_{a m, 1}(z)}{N_{m, 2}(z)-N_{a}(z) C_{a m, 2}(z)}=\frac{f_{m, 1}(T(z), P(z))}{f_{m, 2}(T(z), P(z))}
$$

\section{ATMOSPHERIC TEMPERATURE MEASUREMENT RESULTS}

The atmospheric temperature profile measured on February $19^{\text {th }}, 2017$ from 0:00 to 5:00 UTC Madison, WI, and radiosonde observations from Green Bay, WI, are shown in Figure 2a. The vertical resolution is 300 meters. The difference of the measured profile and the radiosonde is shown in Figure 2b. For the profiles' correction the ratio of the signals in the molecular channels is measured with removed Argon buffered iodine filter. Then, the ratio is applied to the signals in one of the channels. A case for atmospheric temperature profile with inversion layer at the cloud top is shown in Figure 2c (February $15^{\text {th }}$, 2017 3:00-5:00UTC Madison, WI, and radiosonde Green Bay, WI, 150 meters vertical resolution). The site elevation is 330 meters. The HSRL measured profiles differ from the radiosonde observations in the lower $2 \mathrm{~km}$. This difference is partially due to different physical locations of radiosonde and the lidar observations. Also, the earlier described optical scrambler is used to minimize the alignment errors. However, a residual range/time dependency error remains in the signals. We assume it is caused by insufficient optical scrambling, and the detectors' nonlinear response in presence of the large internally scattered laser pulse. The longer scrambler will be implemented in the future.
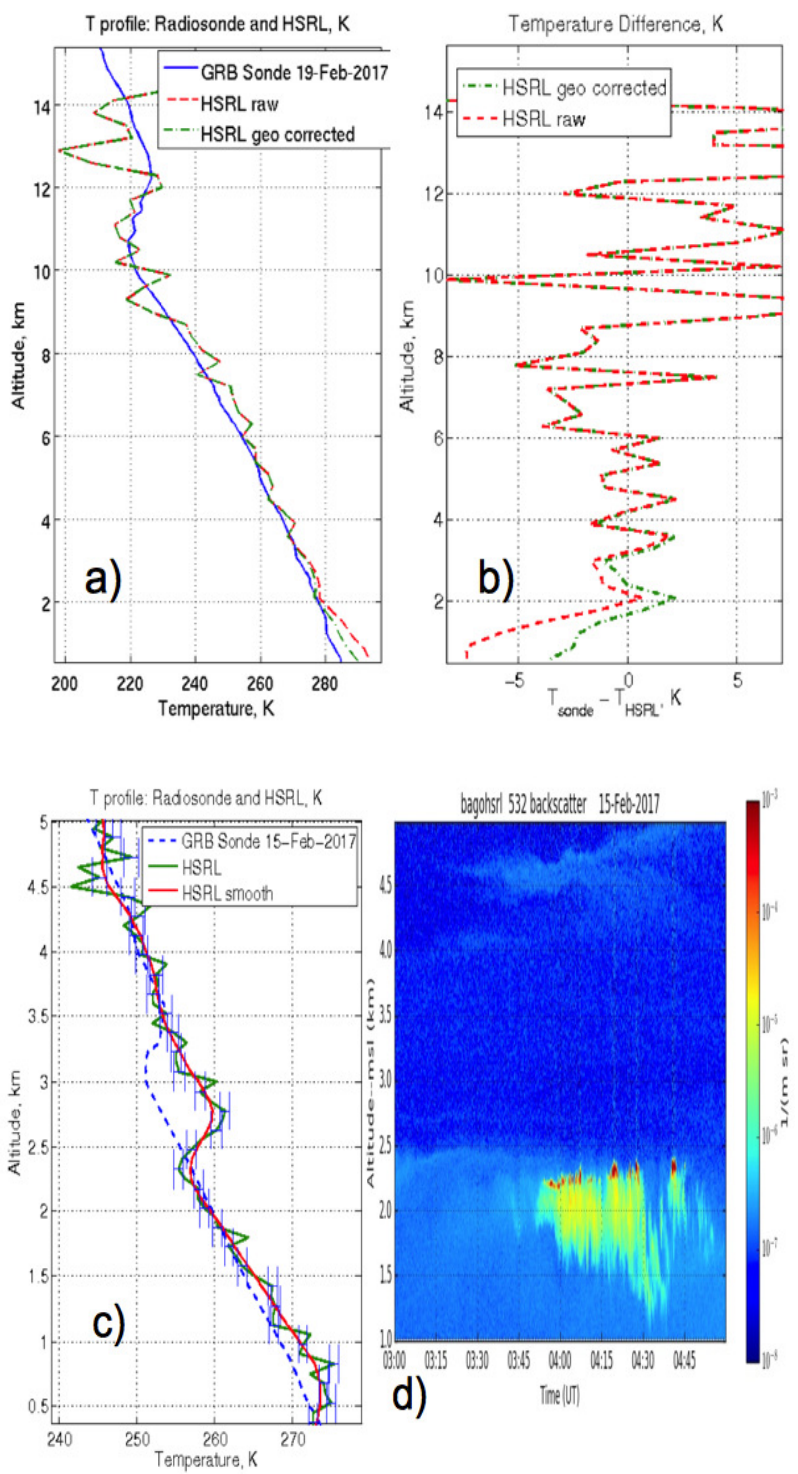

Figure 2. a) Temperature profiles: uncorrected-raw (red, dashed), and corrected (green, dashed); measured on February 19, 2017 from 0:00 to 5:00 UTC Madison, WI; and radiosonde profile (blue, solid) 0:00UTC Green Bay, WI; b) The difference of lidar and radiosonde profiles for uncorrected (red, dashed) and corrected profiles (green, dashed);

c) Temperature profile at $150 \mathrm{~m}$ range resolution (green, solid), smoothed profile at $75 \mathrm{~m}$ resolution (red, solid) on February 15, 2017 from 3:00 to 5:00 UTC Madison, WI; and radiosonde (blue, dashed) OUTC Green Bay, WI; d) Range-time image for aerosol volume backscatter coefficient, $1 /\left(\begin{array}{ll}\mathrm{m} & \mathrm{s}\end{array}\right)$, corresponding to the temperature profile in Fig.c.

The HSRL is co-located with Atmospheric Emitted Radiance Interferometer instrument (AERI), which is capable of measuring the 
atmospheric temperature profile in the lower $4 \mathrm{~km}$ with $\sim 200 \mathrm{~m}$ spatial resolution. This will be used for validation and for correction of the HSRL temperature profiles.

\section{MEASUREMENT SENSITIVITY AND ERROR ANALYSIS}

The temperature profile measured by the HSRL still exhibits an offset between around -5 and +5 $\mathrm{K}$ when compared with the radiosonde sounding. A correction on the relative gain of the two molecular channels must be applied for its correction. In order to explain the temperature offsets, we analyzed the sensitivity of the described temperature profile measurement method to the instrument calibrations. Some of the temperature measurement errors, instrument parameters, and calibration values are summarized in Table. 1.

Table 1 HSRL calibration and measurement errors.

\begin{tabular}{|c|c|c|c|}
\hline Source & Estimate & $\begin{array}{c}\text { Percent } \\
\text { error in } \\
\text { Signal } \\
\text { Ratio }\end{array}$ & $\begin{array}{c}\text { Temperature } \\
\text { Error } \\
(.145 \% / K)\end{array}$ \\
\hline Laser Spectral purity & $1: 5000$ & 0.001 & $\begin{array}{l}<0.01 \mathrm{~K} \\
\text { (clear air) }\end{array}$ \\
\hline $\begin{array}{l}\text { Transmitted Spectral } \\
\text { width }\end{array}$ & $30 \mathrm{MHz}$ & 0.10 & $.7 \mathrm{~K}$ \\
\hline $\begin{array}{c}\text { Frequency Lock } \\
\text { Accuracy }\end{array}$ & $\pm 30 \mathrm{MHz}$ & 1.1 & $\pm 7.5 \mathrm{~K}$ \\
\hline Etalon Drift Stability & $\pm 20 \mathrm{MHz}$ & 0.08 & $\pm 0.6 \mathrm{~K}$ \\
\hline $\begin{array}{l}\text { Iodine filters' } \\
\text { transmission (scan- } \\
\text { to-scan variation) }\end{array}$ & $20 \mathrm{MHz}$ & 0.29 & $\pm 2 \mathrm{~K}$ \\
\hline $\begin{array}{l}\text { Detector gain } \\
\text { stability and } \\
\text { transmission } \\
\text { functions }\end{array}$ & $<0.45 \%$ & $<0.45$ & $3.1 \mathrm{k}$ \\
\hline $\begin{array}{l}\text { Statistical Noise at } \\
\text { different ranges for } \\
\text { 1-hr and 300-m } \\
\text { integration }\end{array}$ & $\begin{array}{l}\text { at } 1 \mathrm{~km} \\
\text { at } 5 \mathrm{~km} \\
\text { at } 10 \mathrm{~km} \\
\text { at } 15 \mathrm{~km}\end{array}$ & $\begin{array}{l}.04 \\
.1 \\
.3 \\
.7\end{array}$ & $\begin{array}{l}0.3 \mathrm{~K} \\
0.6 \mathrm{~K} \\
2.0 \mathrm{~K} \\
4.7 \mathrm{~K}\end{array}$ \\
\hline
\end{tabular}

The largest error is due to the frequency locking accuracy. The laser center frequency during the instrument operation can remain stable within 1 $\mathrm{MHz}$ for several hours. However, under instrument thermal disturbances, the center frequency experiences the offset up to $30 \mathrm{MHz}$ from the peak absorption of the iodine line. Further improvements in the absolute frequency locking stability $(\sim 1 \mathrm{MHz})$ are required in order to decrease the offset to $\sim 0.1 \mathrm{~K}$. The calibration scans measurement error in the channels' transmission is another source of the temperature offsets. The transmissions are corrected by adjusting the relative gain of the channels. The correction remains constant if no changes in the transmission spectrum occur (i.e. etalon drift, iodine filters changes). The correction by LED pulses is used to adjust the channels' transmissions with around $0.01 \%$ accuracy. However, this technique requires more testing to confirm its efficiency.

\section{CONCLUSIONS}

The recent instrument modifications have improved the temperature measurement accuracy. However, it still requires a range correction in the lower $2 \mathrm{~km}$ and a precise laser frequency locking. In the near future we will improve the laser frequency locking accuracy by implementing an interferometer-based laser cavity controller. The future instrument improvements will make the temperature profile measurements robust and useful for meteorological applications.

\section{ACKNOWLEDGEMENTS}

This work is supported by the Space Science and Engineering Center U. of Wisconsin - Madison.

\section{References}

[1] Eloranta, E. W.: High Spectral Resolution Lidar, in Lidar: Range-Resolved Optical Remote Sensing of the Atmosphere, Klaus Weitkamp ed., Springer, NY, pp. 25-30.

[2] Hair J.W. et al, 2001: High spectral resolution lidar with iodine-vapor filters: measurement of atmospheric-state and aerosol profiles, Applied Optics, Vol. 40, Issue 30, pp. 5280-5294.

[3] Liu Z-S. et al 2009: Iodine-filter-based high spectral resolution lidar for atmospheric temperature measurements, Optics Letters, Vol. 34, Issue 18, pp. 2712-2714.

[4] Razenkov Ilya et al, 2015: Atmospheric temperature profile measurements performed by the University of Wisconsin-Madison High Spectral Resolution Lidar, Conference papers ILRC27.

[5] Razenkov Ilya et al, 2017: University of Wisconsin-Madison High Spectral Resolution Lidar, Conference papers ILRC28. 